\title{
DEVELOPMENT OF AN ACTIVE HELICAL DRIVE SELF-BALANCING IN-PIPE ROBOT BASED ON COMPOUND PLANETARY GEARING
}

\author{
Tao Ren, ${ }^{*}$ Yin Zhang, ${ }^{*}$ Yujia Li, ${ }^{* *}$ and Lin Xian*
}

\begin{abstract}
As pipelines increase in age, the required number of pipeline inspections and maintenance also increases on a yearly basis. Regular inspection and maintenance of pipelines using in-pipe robots can eliminate safety hazards in time and ensure production safety. The current study proposes an active helical drive self-balancing in-pipe robot that is based on compound planetary gearing. The design of the driving and supporting systems is described. The gear ratio and obstacle negotiating of the robot are analysed. Traction experiment of the prototype is conducted to demonstrate the effectiveness of the proposed structure. Within the same length, the proposed configuration can be connected in series to a multi-section drive system. The presented in-pipe robot has larger traction force than an active helical drive support-balanced in-pipe robot. The robot can conduct further inspections and implement repair tools to complete pipeline inspection and maintenance work simultaneously.
\end{abstract}

\section{Key Words}

Self-balancing, active helical, in-pipe robot, compound planetary gearing

\section{Introduction}

As pipelines increase in age, the required number of pipeline inspections and maintenance also increases on a yearly basis. Achieving full coverage is difficult for conventional external inspection due to the particularity of pipeline structures. Therefore, using in-pipe robots offers a unique advantage [1]-[3]. With requirements such as different environments and operating conditions, in-pipe

* Robotics Research Center, Xihua University, Chengdu 610039, China; e-mail: rtone@foxmail.com, zyin_94@163.com, xianlin0518@foxmail.com

** School of Mechatronic Engineering, and Post-Doctoral Research Station, Southwest Petroleum University, Chengdu 610500, China; e-mail: yujiali321@foxmail.com

Corresponding author: Yujia Li

Recommended by Prof. Anmin Zhu

(DOI: 10.2316/J.2019.206-0211) robots with various driving methods have been developed [4]-[7]. At the end of the 20th century, companies and scholars studied in-pipe robots for oil and gas wells with increased driving force [8]. However, these pipeline robots are lengthy and thus have a limited scope of use.

Helical in-pipe robots possess certain advantages, such as simple control structure, high reliability and good practicability; thus, they have received increasing attention from scholars [9]-[11]. The helical motion of the in-pipe robot can be achieved through several means, including the conventional passive driving scheme, where the rotation of the wheel carrier drives the driving wheel to passively rotate. The driving wheel generates an axial pulling force that drives the robot to move forward through passive rotation [12], [13]. Another example is the active driving scheme proposed by the authors, where the power source directly propels the driving wheel to rotate and then drives the wheel frame to rotate [14]-[16]. The traction force of the robot is considerably increased relative to the conventional passive helical in-pipe robot and is thus more suitable for hooking detection and repair tools when applied to pipelines.

The aforementioned helical in-pipe robots require a stator (supporting system) to balance the reverse torque generated by the rotor and thereby prevent the robots from rotating on their own axis in the pipe [17]-[21]. The stator increases the length and reduces the passing performance of the robot in pipes with small bend radii and deformities. One way to balance the reverse torque is to drive two helical driving modules with equal speed and reverse rotation. When the motor is electrified, the two rotating modules produce driving forces in the same direction to make the robot move [22]-[24].

However, the wheels of the aforementioned in-pipe robots are driven by the wheel carrier passively relying on the friction force exerted by the pipe wall on the wheels. To obtain a large propulsion, the authors proposed an actively driven spiral pipeline robot on the basis of compound planetary gearing in previous works. A supporting system was designed to balance the counter torque [25]. 


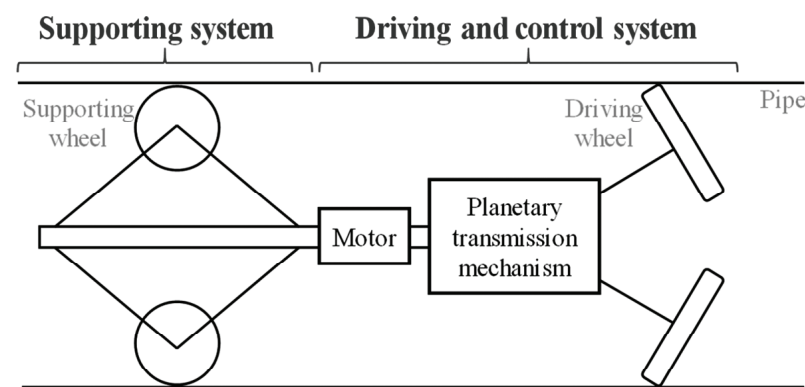

Figure 1. System composition of the active helical drive in-pipe robot.

The current study presents an active helical drive self-balancing in-pipe robot that is based on compound planetary gearing and does not require a supporting system to balance the counter torque. Such property reduces the robot's length. Within the same length, the active helical drive self-balancing in-pipe robot can be connected in series to a multi-section driving system. The presented in-pipe robot has more traction force than an active helical drive support-balanced in-pipe robot. This setup brings notable benefits. The robot can conduct further inspections and implement repair tools to complete pipeline inspection and maintenance work simultaneously.

\section{Driving Principle of the Active Helical Drive Self-balancing In-Pipe Robot}

\subsection{Design of the Driving System}

The driving wheel of the active helical drive in-pipe robot is directly driven by the power output from the motor and transmission system. Figure 1 shows that the power output from the motor is shunted by the planetary transmission mechanism to thrust the driving wheels to rotate. The driving wheel actively rotates when it comes into contact with the inner wall of the pipe because it has a certain angle with the pipe axis. The driving wheels of the robot produce a helical motion on the inner wall of the pipe. The supporting system is designed to serve two primary purposes: to prevent the robot from spinning and to provide a second anchor point so that the robot can be properly centralised inside the pipe. The control system is used to control the moving speed of the robot by controlling the rotational velocity of the motor.

The torque output by the driving motor is directly distributed to each driving wheel through mechanical transmission. When the driving motor exports the torque, it generates a reverse torque. According to the reverse torque balancing structure, the active helical drive in-pipe robot can be divided into an active helical drive supportbalanced in-pipe robot (Fig. 2a) and an active helical drive self-balancing in-pipe robot (Fig. 2b).

Figure 2(a) shows that the motor shell of the supportbalanced transmission structure is fixed to the supporting structure. The counter torque is generated by the motor body and transmitted to the supporting structure through the motor shell. The friction between the guiding wheels

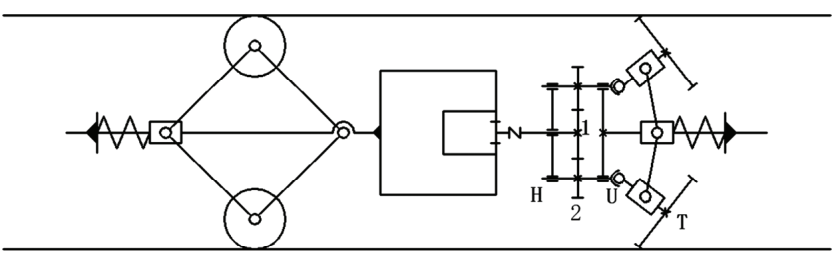

(a)

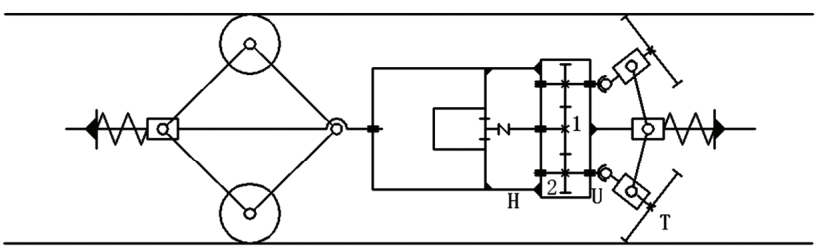

(b)

Figure 2. Transmission structure of the active helical drive in-pipe robot: (a) support-balanced transmission structure and (b) self-balancing transmission structure.

and pipe wall is balanced by the counter torque of the motor. This structure realises the engagement of the secondstage planetary gear set through the mesh of the driving wheel to the inner wall of the pipe. This framework is a remarkable feature of the active helical drive in-pipe robot.

Figure 2(b) depicts the self-balancing transmission structure proposed in this study. The motor remains mounted in the motor shell, which is rotatable coupled to the support structure to transmit only axial tension without transmitting torque. The motor output shaft is connected to gear 1 that meshes with gear 2 , which in turn is mounted on the planet carrier H. This carrier is fixedly connected to the motor shell. Gear 2 connects driving wheel $\mathrm{T}$ through universal joint $\mathrm{U}$. The driving wheels mesh the inner wall of the pipe. Finally, the reverse torque of the motor is balanced by the overall structure without requiring a supporting structure.

\subsection{Transmission Ratio Analysis}

The transmission ratio amongst the various structures of a robot is the main factor that affects the performance of the driving system. Table 1 presents the structural parameters in Fig. 3.

Table 1

Parameters of Active Helical Drive Self-balancing In-Pipe Robot

\begin{tabular}{|l|c|c|}
\hline Parameters & Radius & Rotary Speed \\
\hline Motor M & & $\omega$ \\
\hline Reference circle of gear 1 & $r 1$ & $\omega_{1}$ \\
\hline Reference circle of gear 2 & $r 2$ & $\omega_{2}$ \\
\hline Drive wheel T & $r_{\mathrm{T}}$ & $\omega_{\mathrm{r}}$ \\
\hline Planet carrier H & & $\omega_{\mathrm{H}}$ \\
\hline Pipe radius & $R$ & \\
\hline
\end{tabular}




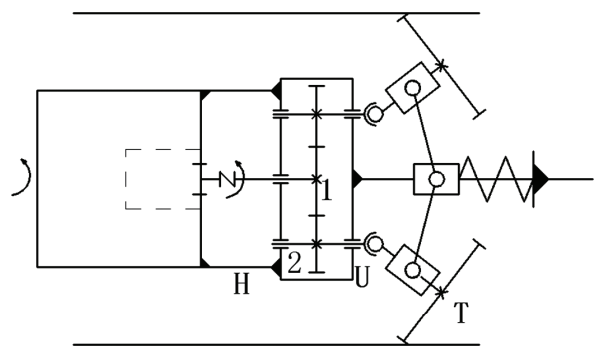

Figure 3. Self-balancing transmission structure.

A common angular velocity $-\omega_{\mathrm{H}}$, which is the same as the angular velocity of planet carrier $\mathrm{H}$ and in the opposite direction, is added. We must ensure that a relative rotation does not exist between the planet carrier and the pipe. The driving wheel $\mathrm{T}$ is ignored in the meantime.

Currently, the motor and carrier $\mathrm{H}$ do not rotate relative to each other, and the output shaft of the motor is connected to gear 1 . Therefore, we obtain:

$$
\omega_{1}^{H}=\omega
$$

The rotational speed remains at $\omega_{2}^{H}=\omega_{2}$ after the common angular velocity is virtually exerted because the rotation centre of gear 2 does not coincide with the rotation centre of the pipe. From the meshing conditions, we can obtain:

$$
\omega_{1}^{H} r_{1}=\omega_{2}^{H} r_{2}
$$

Remove the virtual common angular velocity $-\omega_{\mathrm{H}}$. The movement of gears 1 and 2 is transmitted to the remainder of the structure. At the point of contact, a circumferential relative slip does not exist between the driving wheels and the inner pipe wall. Thus, we have:

$$
\omega_{H} r_{4}=\omega_{3} r_{3}
$$

Simultaneous to (1)-(3), the transmission ratio of the motor to the driving wheel frame can be obtained:

$$
i_{M H}=\frac{\omega}{\omega_{H}}=\frac{r_{2} R}{r_{1} r_{T}}
$$

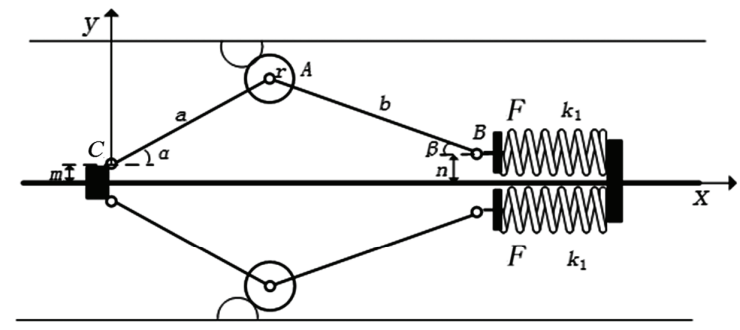

(a)

\section{Design of the Supporting System}

The central axis of the in-pipe robot is as close as possible to the axis of the pipeline through the supporting system to prevent the robot from being considerably hindered due to axis deviation. Therefore, whether the structural design of the supporting system is reasonable determines the negotiating performance of the in-pipe robot. Balancing the reverse torque is not required for the active helical drive self-balancing in-pipe robot proposed in this work. Hence, spring support can be used to meet the requirements.

When the conventional single support spring exerts force, the movement of each support arm is intertwined [26], [27]. The supporting system of the proposed inpipe robot adopts the arrangement of parallel compression springs. Figure 4 shows that one end of the support arm is hingedly fixed to the central shaft, whereas the other end is hinged to the adjustment slider. The adjustment slider is fitted with an adjustment spring. For ease of analysis, we assume that the support unit has only two upper and lower symmetrical support arms.

The fixed end on the left side of the support arm is taken as the coordinate origin, and the horizontal and vertical directions are the $x$ - and $y$-axes, respectively. Figure 4 establishes the Cartesian coordinate system and defines each parameter. The preload of the spring is $2 F$. Therefore, we obtain the following:

$$
\left\{\begin{array}{l}
y_{A}=m+a \sin \alpha \\
x_{B}=a \cos \alpha+b \cos \beta \\
m+a \sin \alpha=n+b \sin \beta
\end{array}\right.
$$

During field operations, in-pipe robots encounter a variety of obstacles, the majority of which are annular (Fig. 4a) and unilateral (Fig. 4b) obstacles. The annular obstacle in general refers to the gap between two pipes, elbow and branch of the pipes, whereas unilateral obstacles are in general referred to as local damage and barriers in the pipe.

Figure 4 a shows that the robot encounters an annular obstacle as it moves in the pipeline. Let us assume that the distance between the wheel and the inner wall of the pipe is $\Delta$. When the support wheel passes the obstacle, the contact position between the wheel and the obstacle changes in real time. The force direction is not in the

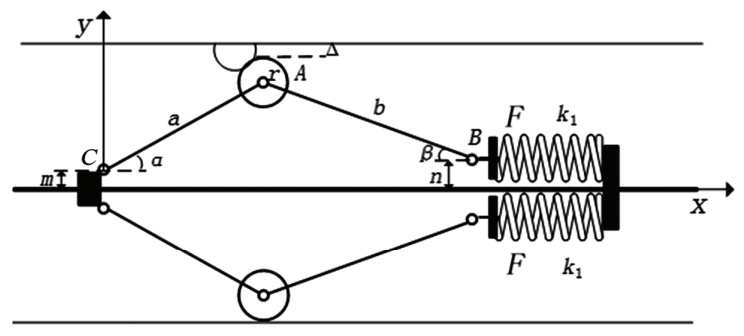

(b)

Figure 4. Support arms encounter barriers: (a) annular and (b) unilateral obstacles. 


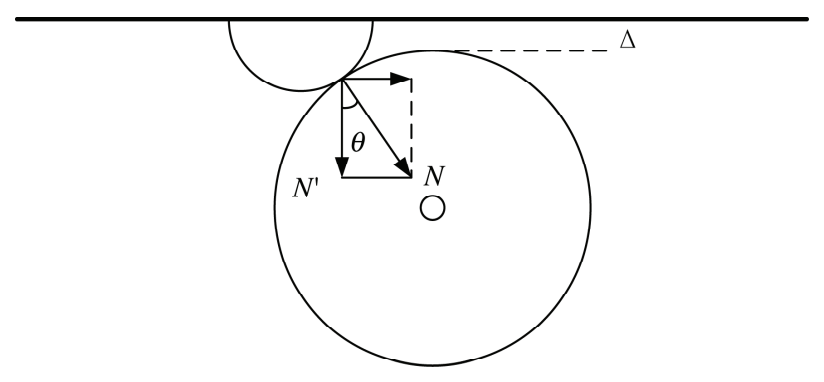

Figure 5. Force condition of the supporting wheel when in contact with an obstacle.

radial direction of the pipe but at an angle $\theta$ to the radial direction, as shown in Fig. 5. The spring force on one of the support arms is denoted as $F_{1}$.

From the principle of virtual work, we obtain:

$$
N^{\prime} \delta y_{A}+F_{1} \delta x_{B}=0
$$

The change in spring compression is:

$$
\Delta L_{1}=(a \cos \alpha+b \cos \beta)-\left(a \cos \alpha_{1}+b \cos \beta_{1}\right)
$$

The force at which the spring acts on one of the support arms is:

$$
F_{1}=F+k_{1} \Delta L_{1}
$$

The relationship between $F$ and $N$ can be obtained as:

$$
N=\frac{\left(F+k_{1} \Delta L_{1}\right)\left(\tan \alpha_{1}+\tan \beta_{1}\right)}{\cos \theta}
$$

As shown in Fig. 4b, when the parallel spring encounters a single-sided obstacle, the relationship between $N$ and $F$ becomes:

$$
N=\frac{\left(F+k_{1} \Delta L_{2}\right)\left(\tan \alpha_{2}+\tan \beta_{2}\right)}{\cos \theta}
$$

Table 2

Comparison of Two Support Structures

\begin{tabular}{|l|c|c|}
\hline Force & Single Support & Parallel Support \\
\hline $\begin{array}{l}\text { Annular obstacle } \\
\text { supporting force }\end{array}$ & $N=\frac{\left(2 F+k \Delta L_{1}\right)\left(\tan \alpha_{1}+\tan \beta_{1}\right)}{2 \cos \theta}$ & $N=\frac{\left(F+k_{1} \Delta L_{1}\right)\left(\tan \alpha_{1}+\tan \beta_{1}\right)}{\cos \theta}$ \\
\hline $\begin{array}{l}\text { Unilateral obstacle } \\
\text { supporting force }\end{array}$ & $N=\frac{\left(2 F+k \Delta L_{2}\right)\left(\tan \alpha_{2}+\tan \beta_{2}\right)}{2 \cos \theta}$ & $N=\frac{\left(F+k_{1} \Delta L_{2}\right)\left(\tan \alpha_{2}+\tan \beta_{2}\right)}{\cos \theta}$ \\
\hline $\begin{array}{l}\text { Force from } \\
\text { unilateral obstacle } \\
\text { to central axis }\end{array}$ & $N=\left(2 F+k \Delta L_{2}\right)\left(\tan \alpha_{2}+\tan \beta_{2}\right)$ & $F_{z}=F\left(\tan \alpha_{2}+\tan \beta_{2}-\tan \alpha-\tan \beta\right)$ \\
\hline
\end{tabular}




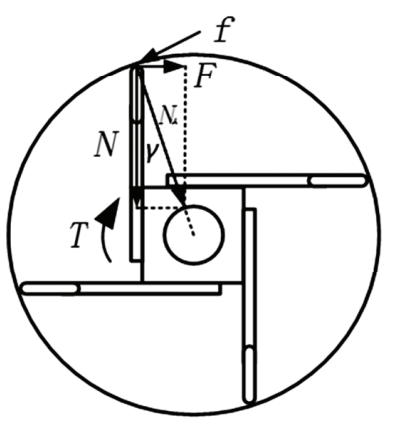

(a)

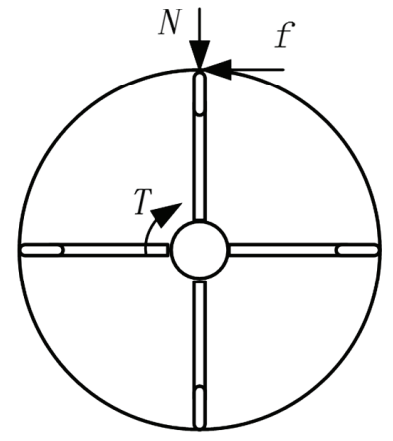

(b)
Figure 6. Mechanism of the supporting arms: (a) eccentric type and (b) symmetrical type.

to the torque balance of the system, the stable condition of the robot is:

$$
\frac{4 N \mu R}{\cos \gamma} \geq T
$$

However, the active helical drive in-pipe robot presented in this paper is self-balancing type and does not require an eccentric mechanism to balance the reverse torque. Therefore, the supporting system of the robot is designed as a symmetrical mechanism.

Similarly, the stable condition of the four-symmetrical arm structure is:

$$
4 N \mu R \geq T
$$

An angle $\gamma$ exists between the vertical and resultant forces of the supporting wheel of the eccentric supporting mechanism, which can amplify the positive pressure of the supporting arm and thus can withstand a large reverse torque. For in-pipe robots that do not require to balance the reverse torque, only the symmetrical supporting mechanism needs to be used.

In the eccentric supporting mechanism shown in Fig. 7, the contraction-expansion of the supporting arms and the spring pretension are controlled by manually rotating the spring clamp. If the robot encounters a large obstacle in the pipeline or the pipe radii changes greatly, the supporting arms can only be moved by the slight deformation of the spring. Robots are more likely to get stuck in the pipeline and cannot move forward.

To ensure the negotiating capacity of the active screw drive in-pipe robot in the case of large changes in the pipeline environment, the supporting mechanism is optimised in this study. As shown in Fig. 8, the automatic expansion and contraction of the supporting arms are realised by controlling the movement of the ball screw. The force sensors are mounted to the end of the supporting arms. When the environment inside the pipe changes, the supporting force exerted on the supporting arm by the pipeline also changes. The data of the force sensor are fed back to the control system. The system controls the motor to automatically adjust the expansion and contraction of

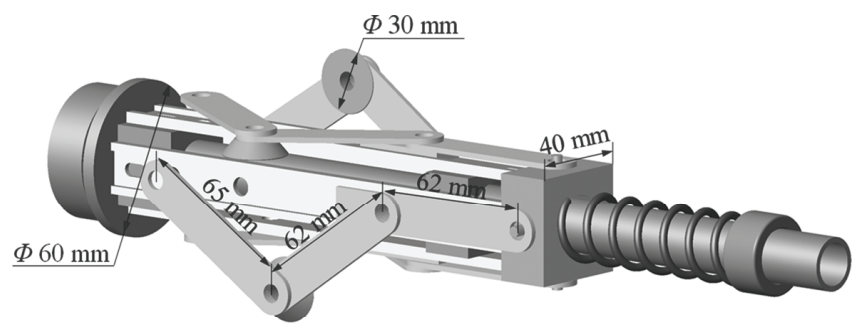

Figure 7. Engineering design drawing of the eccentric supporting mechanism.

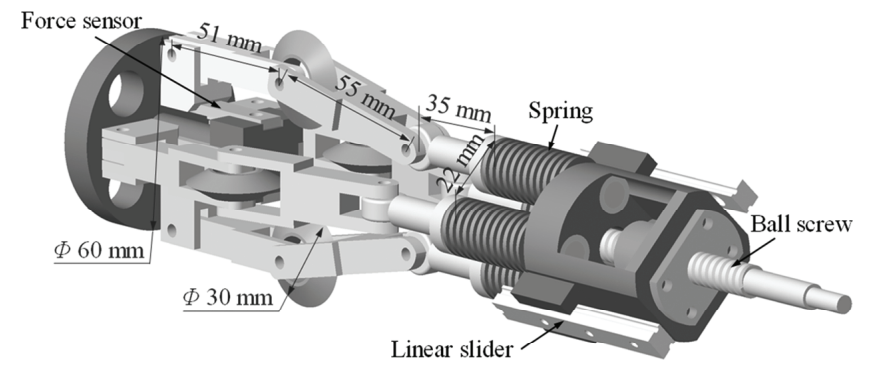

Figure 8. Engineering design drawing of the symmetrical supporting mechanism.

the supporting arm to achieve a constant supporting force and ensure the negotiating capacity of the in-pipe robot.

\section{Traction Experiment of the Active Helical Drive Self-balancing In-Pipe Robot Prototype}

\subsection{Prototype}

An adjustment mechanism for controlling the extension arm is fabricated to form a self-balancing modular unit. The drive unit of the in-pipe robot has a diameter of $74 \mathrm{~mm}$ and a length of $900 \mathrm{~mm}$. The drive motor is a DC motor at $200 \mathrm{~W}$ and $170 \mathrm{rpm}$. The telescopic motor of the driving wheel is a DC motor at $30 \mathrm{~W}$ and $20 \mathrm{rpm}$. The support unit adopts a four-arm structure, and the telescopic motor of the supporting wheel is a DC motor at $30 \mathrm{~W}$ and $20 \mathrm{rpm}$.

Figure 9 shows that the prototype of the modular active helical drive self-balancing in-pipe robot consists of driving, supporting and control units. Figure 10 shows a prototype of the driving unit of the modular active helical drive self-balancing in-pipe robot, where the drive motor directly drives the sun gear, which meshes with the first planetary gearing set. The second planetary gearing set is connected to the output shaft of the first planetary gearing set through a telescopic universal joint. The inner wall of the pipe acts as the inner ring gear of the planetary gear system. The ball screw is driven by the wheel telescopic motor. The linear slider bears the reverse torque of the screw nut, which drives the adjustment unit to push or retract the link to control the contraction and extension of the driving wheels. The compression spring between the linear slider and the screw nut adapts the robot to a range of pipe diameter changes. The drive motor and control circuit are fixed to the body of the in-pipe robot and rotate 


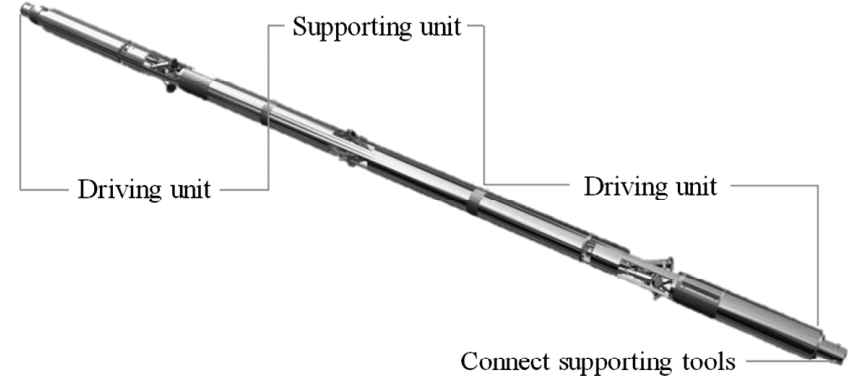

Figure 9. Prototype of the modular active helical drive self-balancing in-pipe robot.

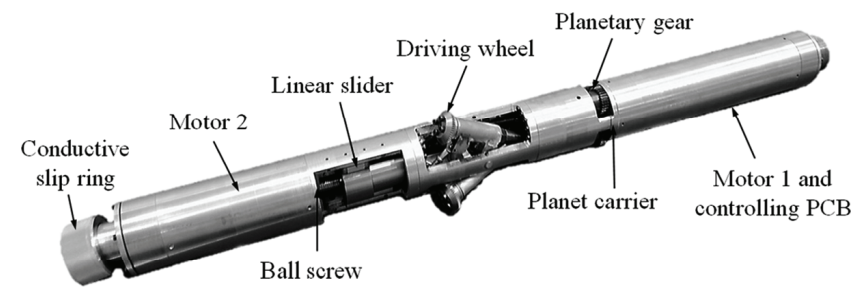

Figure 10. Driving unit prototype.

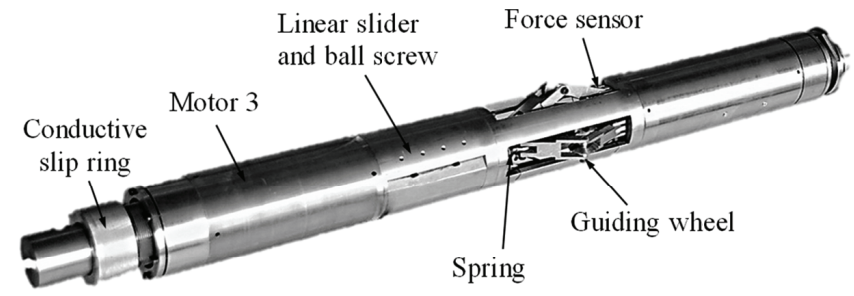

Figure 11. Supporting unit prototype.

with the planet carrier. In this configuration, the force amongst all the transmission components is the internal force of the system, and no external structure is required to achieve a balance.

The supporting unit can be added in the modular helical in-pipe robot system. It is used only to improve the negotiating performance and motion stability of the robot in the pipeline without balancing the reverse torque. Figure 11 displays the prototype of the supporting unit. A strain gauge force sensor is added to one end of the four supporting wheels to test their positive pressure against the pipe wall.

The internal control signals and power supply system should communicate and supply power amongst the relatively rotating unit modules due to the relative rotation between the individual unit modules of the modular selfbalancing in-pipe robot. Therefore, a conductive slip ring is disposed between adjacent modules. Power carrier communication is used in the modular self-balancing in-pipe robot. To reduce power loss in the transmission line, we increase the supply voltage to $110 \mathrm{~V}$ DC. The power carrier is also used in the communication between the module and the control software and between the two modules. The carrier code rate is set to $512 \mathrm{kbps}$.

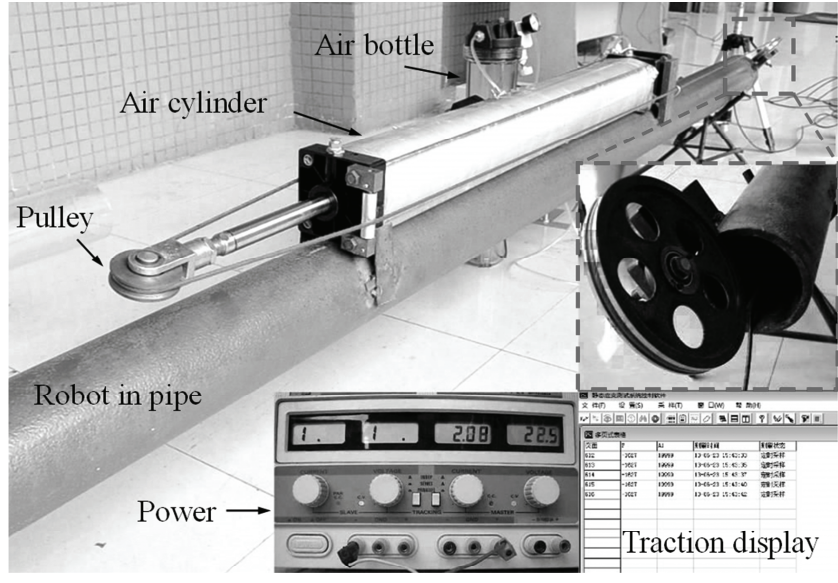

Figure 12. Experiment of the active helical drive selfbalancing in-pipe robot.

\subsection{Experiments}

A seamless steel pipe with an outer diameter of $127 \mathrm{~mm}$, wall thickness of $5 \mathrm{~mm}$ and length of $9 \mathrm{~m}$ is used in the traction test of the modular active helical drive self-balancing in-pipe robot. A robot equipped with driving and supporting units moves forward in the pipeline. A wire rope is connected to the tail of the robot, and the wire rope is guided by two pulleys, which are connected to the force sensor. Figure 12 illustrates the experimental device.

The output thrust of the cylinder can be changed by adjusting the gas pressure in the cylinder. The robot and cylinder output are connected by a wire rope to test the different traction forces of the robot. The external DC power supply provides power to the robot system. The power carrier is used to transmit signals through the power supply cable in the control system. The traction force of the robot can be obtained in real time through an external force sensor.

The robot moves forward in the pipeline. As the pressure of the air in the cylinder gradually increases, the pulling force that acts on the in-pipe robot increases, as shown in Fig. 13. When the driving wheel of the robot slips and no longer moves forward, the stable pulling force at this time is considered the maximum traction force of the in-pipe robot. The maximum traction force of the inpipe robot is tested in the experiment to be $1,620 \mathrm{~N}$. The electric voltage and current are recorded, and the input power is $160 \mathrm{~W}$. The rotational speed of the driving motor is measured by an encoder to be $170 \mathrm{rpm}$. In a pipe with an outer diameter of $127 \mathrm{~mm}$, the helical angle of the robot driving wheel is $15^{\circ}$. According to the calculation of motor torque and gear ratio from (1) to (4) and the force balance relationship of the driving wheels, the theoretical value of traction force is $5,413 \mathrm{~N}$. The mechanical efficiency of the transmission system is approximately $30 \%$. The efficiency of the robot driving motor to convert electrical energy into mechanical energy is approximately $86 \%$. The transmission efficiency of the motor planetary gear reducer is $65 \%$ according to the motor selection manual. The robot's two-stage planetary gear train has a transmission efficiency 


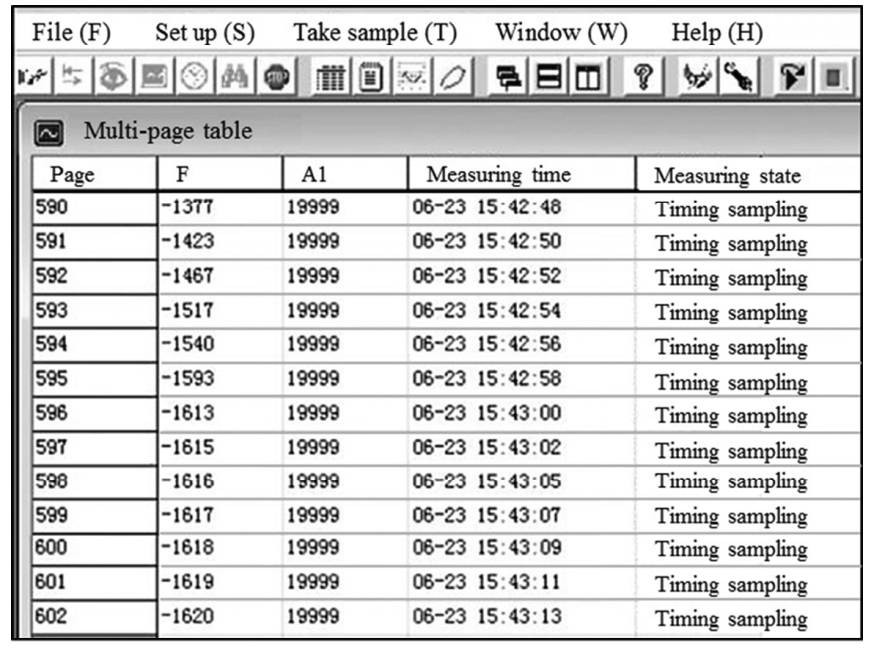

Figure 13. Experimental traction force and current of the in-pipe robot.

of $81 \%$. In summary, the total efficiency of the system is 45.3\%. In addition, many uncalculated friction forces are still present in the system, which will increase the power loss and reduce the transmission efficiency. Finally, the transmission efficiency of the in-pipe robot approximates the calculated value.

The performance test of a single driving unit proves that the in-pipe robot has a strong traction capability. In actual use, the robot can be connected to multiple tools to suit different job requirements.

\section{Conclusion}

This article presents an active helical drive self-balancing in-pipe robot that is based on compound planetary gearing. Unlike most existing helical drive in-pipe robots reported previously, this newly designed in-pipe robot does not require a supporting system to balance the counter torque. Such property reduces the robot's length. Within the same length, the proposed configuration can be connected in series to a multi-section drive system. The presented in-pipe robot has more traction force than an active helical drive support-balanced in-pipe robot.

The transmission ratio amongst the various structures of the proposed robot mechanism is calculated to analyse the performance of the driving system. The supporting system of the in-pipe robot adopts the arrangement of parallel compression springs. The force on the central shaft of the parallel compression springs is less than that of the single-spring support when the parallel spring is supported by a unilateral obstacle. The modular prototype of the active helical drive self-balancing in-pipe robot is fabricated. Conductive slip ring and power carrier communication are used to ensure the communication and power supplementary amongst the relatively rotating unit modules of the modular self-balancing in-pipe robot. The maximum traction force of the prototype is $1,620 \mathrm{~N}$.

Future work will focus on adding pipeline detection module to the modular prototype for verifying the feasibility of the proposed in-pipe robot in pipeline inspection work.

\section{Acknowledgements}

This work was supported in part by National Natural Science Foundation of China (NSFC) under Project 51805443, Sichuan Science and Technology Program under Project 2019YFG0123 and 2019YFG0336, Young Scholar and Young Scholar for Reserve Talents of Xihua University.

\section{References}

[1] Y. Li, Q. Liu, Y. Chen, and M. Li, Helical-contact deformation measuring method in oil-gas pipelines, International Journal of Robotics and Automation, 32(1), 2017, 55-62.

[2] A. Shukla and H. Karki, Application of robotics in onshore oil and gas industry - A review Part I, Robotics and Autonomous Systems, 75, 2016, 490-507.

[3] S. Datta and S. Sarkar, A review on different pipeline fault detection methods, Journal of Loss Prevention in the Process Industries, 41, 2016, 97-106.

[4] T. Mirats, M. Josep, and W. Garthwaite, Robotic devices for water main in-pipe inspection: A survey, Journal of Field Robotics, 27(4), 2010, 491-508.

[5] H.M. Kim, Y.S. Choi, Y.G. Lee, and H.R. Choi, Novel mechanism for in-pipe robot based on a multiaxial differential gear mechanism, IEEE/ASME Transactions on Mechatronics, 22(1), 2017, 227-235.

[6] Y. Zhu, X. Sun, and X. Wang, Locomotion system design and dynamics analysis of a new telescopic miniature in-pipe robot, International Journal of Robotics and Automation, 31(2), 2016, 111-117.

[7] M.A. Fanni, M.G. Alkalla, and A. Mohamed, Propeller-type skid steering climbing robot based on a hybrid actuation system, International Journal of Robotics and Automation, 33(3), 2018, 258-265.

[8] M. Billingham, A.M. El-Toukhy, M.K. Hashem, M. Hassaan, M. Lorente, T. Sheiretov, and M. Loth, Conveyance-down and out in the oil field, Oilfield Review, 23(2), 2011, 18-31.

[9] T. Ren, Q. Liu, Y. Chen, and S. Ji, Variable pitch helical drive in-pipe robot, International Journal of Robotics and Automation, 31(3), 2016, 263-271.

[10] M. Horodinca, I. Doroftei, E. Mignon, and A. Preumont, A simple architecture for in-pipe inspection robots, International Colloquium on Autonomous and Mobile Systems, Stuttgart, DE, 2002, 61-64.

[11] L. Liang, P. Tang, B. Chen, Y. Tang, Y. Xu, and D. Lin, Dynamical modelling and structural parameter optimization of a novel spiral in-pipe robot, International Journal of Robotics and Automation, 31(1), 2016, 16-25.

[12] Q. Liu, T. Ren, and Y. Chen, Characteristic analysis of a novel in-pipe driving robot, Mechatronics, 23(4), 2013, 419-428.

[13] Y. Chen, Q. Liu, and T. Ren, A simple and novel helical drive in-pipe robot, Robotica, 33(4), 2015, 920-932.

[14] T. Ren, Y. Chen, and Q. Liu, A helical drive in-pipe robot based on compound planetary gearing, Advanced Robotics, 28(17), 2014, 1165-1175

[15] T. Ren, Q. Liu, Y. Li, and Y. Chen, Design, analysis and innovation in variable radius active screw in-pipe drive mechanisms, International Journal of Advanced Robotic Systems, 14(3), 2017, 1-9.

[16] Y. Li, Q. Liu, Y. Chen, and T. Ren, Design and analysis of an active helical drive downhole tractor, Chinese Journal of Mechanical Engineering, 2(30), 2017, 428-437.

[17] T. Li, S. Ma, B. Li, M. Wang, Z. Li, and Y. Wang, Development of an in-pipe robot with differential screw angles for curved pipes and vertical straight pipes, Journal of Mechanisms and Robotics, 9(5), 2017, 051014.

[18] P. Li, S. Ma, C. Lyu, X. Jiang, and Y. Liu, Energy-efficient control of a screw-drive pipe robot with consideration of actuator's characteristics, Robotics and Biomimetics, 3(1), 2016, 1-11. 
[19] A. Kakogawa, T. Nishimura, and S. Ma, Development of a screw drive in-pipe robot for passing through bent and branch pipes, IEEE International Symposium on Robotics, Seoul, KP, 2013, $1-6$.

[20] M. Horodinc, I. Doroftei, E. Mignon, and A. Preumont, A simple architecture for in-pipe inspection robots, Int. Colloquium on Mobile and Autonomous Systems, Magdeburg, DE, 2002, $61-64$.

[21] A. Brunete, M. Hernando, and E. Gambao, Modular multiconfigurable architecture for low diameter pipe inspection microrobots, IEEE Conf. on Robotics and Automation (ICRA), Barcelona, ES, 2005, 490-495.

[22] H. Fukushima, S. Satomura, T. Kawai, M. Tanaka, T. Kamegawa, and F. Matsuno, Modeling and control of a snake-like robot using the screw-drive mechanism, IEEE Transactions on Robotics, 28(3), 2012, 541-554.

[23] S. Hirose, H. Ohno, T. Mitsui, and K. Suyama, Design of in-pipe inspection vehicles for $\Phi 25, \Phi 50, \Phi 150$ pipes, IEEE Conf. on Robotics and Automation (ICRA), Detroit, USA, 1999, 2309-2314.

[24] M.E. Rentschler, S.M. Farritor, and K.D. Iagnemma, Mechanical design of robotic in vivo wheeled mobility, Journal of Mechanical Design, 129(10), 2007, 1037-1045.

[25] Q. Liu, T. Ren, and Y. Chen, Actively driven spiral pipeline robot. 2016.4.19. U.S. Patent 9316340.

[26] Y. Li, Q. Liu, and W. Li, Development of a novel oil and gas in-pipe robot, International Journal of Mechatronics and Manufacturing Systems, 8(3-4), 2015, 102-115.

[27] Q. Liu, T. Ren, Y. Li, and Y. Chen, Down-hole robots: current status, challenge and innovation, IEEE Conf. on Mechatronics and Automation (ICMA), Takamatsu, JP, 2013, 1703-1707.

\section{Biographies}

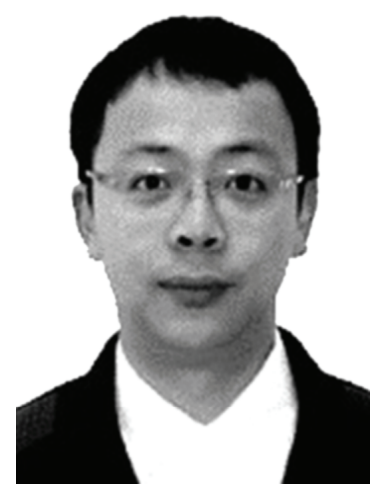

Tao Ren received the B.S. degree in process equipment and control engineering and the M.S. degree in power engineering, and the Ph.D. degree in mechanical engineering from Southwest Petroleum University, Chengdu, China, in 2011, 2014 and 2017, respectively. From 2015 to 2016, he was a Research Associate with the Department of Mechanical Engineering, The University of Hong Kong. He is currently an Associate Professor with the Robotics Research Center, Xihua University, China. His research interests include robotic design and mechatronics systems.

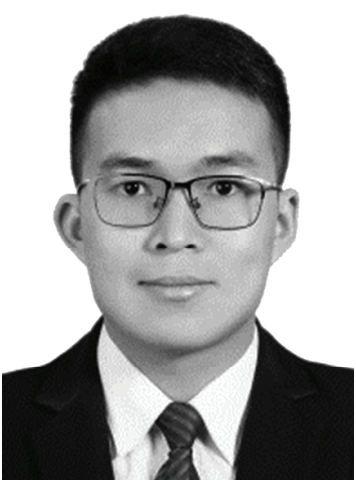

Yin Zhang received the B.S. degree from the School of Intelligent Manufacturing, Panzhihua University, Sichuan Province, China, in 2017. He is currently working toward his M.S. in the School of Mechanical Engineering, Xihua University. His research interests include smart soft robot and in-pipe inspection robot.

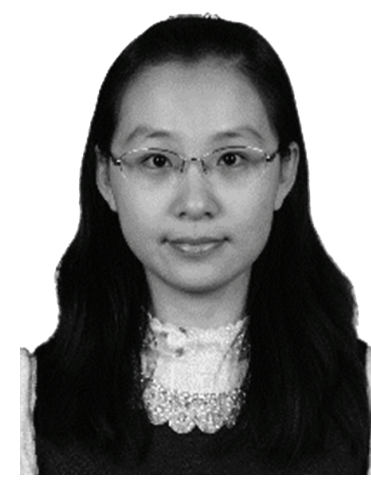

Yujia $L i$ received the B.S. degree in process equipment and control engineering and the Ph.D. degree in mechanical engineering from Southwest Petroleum University, Chengdu, China, in 2012 and 2017, respectively. From 2015 to 2016 , she was a Research Associate with the Department of Mechanical Engineering, The University of Hong Kong. She is currently a Lecturer and a Postdoctoral Fellow with Southwest Petroleum University, China. Her research interests include specialized robot, design and modelling of advanced robotic systems.

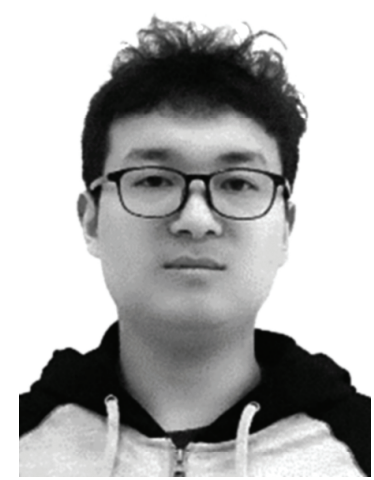

Lin Xian received the B.S. degree from the School of Mechanical Engineering, Xihua University, Sichuan Province, China, in 2018. He is currently working toward his M.S. in the School of Mechanical Engineering, Xihua University. His research interests include control of advanced mechatronics systems. 